\title{
Efecto de la disminución de la velocidad vehicular en la durabilidad de una capa asfáltica
}

\author{
Effect of reducing traffic speed on the durability of a road asphalt layer
}

\author{
Hugo Alexander Rondón Quintana ${ }^{1} \quad$ Fredy Alberto Reyes Lizcano ${ }^{2}$ \\ Carlos Felipe Urazán Bonells ${ }^{3}$
}

Recibido 2 de marzo de 2011, aceptado 13 de noviembre de 2012

Received: March 2, $2011 \quad$ Accepted: November 13, 2012

\begin{abstract}
RESUMEN
Actualmente, en América Latina las vías están experimentando un incremento en el nivel de congestionamiento vehicular en sus vías principales, y con ello una consecuente baja velocidad de marcha. En el artículo se presentan los resultados de una fase experimental desarrollada con el fin de evidenciar el cambio que experimentan las propiedades mecánicas bajo carga repetida de una mezcla densa en caliente tipo MDC-2 cuando se disminuye la velocidad de circulación vehicular. El caso de estudio que se reporta es para mezclas que se fabrican y construyen en Bogotá D.C., Colombia. Como conclusión general se obtiene que al reducir los vehículos la velocidad de circulación sobre una vía en servicio, la mezcla de concreto asfáltico disminuye su rigidez y la resistencia a fatiga. Por ejemplo, disminuir la velocidad de $60 \mathrm{~km} / \mathrm{h}$ a $30 \mathrm{~km} / \mathrm{h}$ genera una disminución aproximada de 15,6\% en la rigidez de la mezcla analizada y $39,7 \%$ en su vida a fatiga.
\end{abstract}

Palabras clave: Módulo resiliente, resistencia a fatiga, mezcla asfáltica densa en caliente, movilidad urbana, velocidad en vías urbanas.

\begin{abstract}
Due to increased congestion on its main routes, the cities of Latin American, have been experiencing a reduction in traffic speed. This article presents the results of research conducted to establish the changes of the mechanical properties under repeated loads that occur in a MDC-2 hot mix asphalt (HMA) when the vehicular speed decreases. The studies were carried out using asphalt mixtures produced in the city of Bogotá, Colombia. As a general conclusion, it has been found that by reducing the speed of traffic, the asphalt concrete mixture reduces its stiffness and resistance to fatigue. For example, a reduction of the speed from $60 \mathrm{~km} / \mathrm{h}$ to $30 \mathrm{~km} / \mathrm{h}$ results in a reduction of approximately $15.6 \%$ in the stiffness of the asphalt mixture and $39.5 \%$ in its fatigue life.
\end{abstract}

Keywords: Resilient modulus, fatigue resistance, dense hot mix asphalt, urban mobility, traffic speed in urban areas.

\section{INTRODUCCIÓN}

La última década del siglo $\mathrm{XX}$ trajo consigo un fuerte incremento en el número de automóviles en circulación en América Latina, implicando que con el paso del tiempo sea más propicia la condición de congestionamiento vehicular, entendido este como "la condición que prevalece si la introducción de un

\footnotetext{
1 Facultad del Medio Ambiente y Recursos Naturales. Universidad Distrital Francisco José de Caldas. Dirección: Vía Circunvalar Venado de Oro. Bogotá D.C., Colombia. E-mail: harondonq@udistrital.edu.co

2 Departamento de Ingeniería Civil. Pontificia Universidad Javeriana. Calle $40 \mathrm{~N}^{\circ}$ 5-50, Edificio José Gabriel Maldonado, Piso 1, Bogotá D.C., Colombia. E-mail: fredy.reyes@ javeriana.edu.co

3 Programa de Ingeniería Civil. Universidad de La Salle. Cra. 2 № 10-70, Sede La Candelaria, Piso 7. Bogotá D.C., Colombia. E-mail: caurazan@unisalle.edu.co
} 
vehículo en un flujo de tránsito aumenta el tiempo de circulación de los demás" [1]. A su vez, a medida que aumenta el tránsito se reduce fuertemente la velocidad de circulación [2].

El caso de la ciudad de Bogotá D.C. (Colombia) no escapa al gran aumento del parque automotor, pues en el año 1999 se matricularon 13.762 vehículos, para luego pasar a la cifra de 113.672 vehículos matriculados en 2006, lo que representa una tasa anual de aproximadamente 12.500 vehículos [3]. No obstante, esa tasa anual se ha multiplicado en más de 10 veces, llegando a registrar tan solo en el año 2010 un monto de 145.571 vehículos nuevos [4]. El Proyecto de Acuerdo citado también expone como causas del congestionamiento vehicular en la actualidad a las obras de mejoras viales.

Como se ha mencionado, el congestionamiento vehicular está ligado a la velocidad de circulación, la cual viene registrando en las arterias de Bogotá D.C. valores en un rango que oscila entre 13 y 30 kilómetros por hora [5], y donde la tendencia de las velocidades más bajas para cada corredor se da para transporte público colectivo en la franja horaria entre las 16:00 y las 20:00 horas. Ese rango de velocidades se traduce en una disminución de más del $50 \%$ del límite de velocidad para vías urbanas en Colombia, el cual está establecido en 60 kilómetros por hora [6].

Dicha disminución de la velocidad vehicular genera cambios en las propiedades mecánicas bajo carga repetida en las mezclas de concreto asfáltico utilizadas para conformar capas asfálticas de estructuras de pavimentos flexibles. En el artículo se presentan los resultados de una fase experimental desarrollada con el fin de evidenciar el cambio que experimentan las propiedades mecánicas de una mezcla densa en caliente tipo MDC-2 (de acuerdo a las especificaciones del Instituto Nacional de Vías [7]) cuando se disminuye la velocidad de circulación vehicular. Las propiedades analizadas fueron el módulo resiliente y la resistencia a fatiga, las cuales son las utilizadas para el dimensionamiento de pavimentos por múltiples métodos de diseño (p.e., [8-11]). Las mezclas fueron fabricadas con un cemento asfáltico (CA) tipo CA 80-100 (PG 52-28) ya que este es el ligante recomendado por INVIAS [7] para construir capas asfálticas en zonas con temperatura media anual promedio inferior a $24{ }^{\circ} \mathrm{C}$ (caso Bogotá D.C.). CA
80-100 se refiere a un cemento asfáltico cuya medida en el ensayo de penetración ASTM D-5 está en un rango entre 80 y 100 décimas de $\mathrm{mm}$.

Con respecto al estado del conocimiento sobre el tema, por lo general la influencia de la velocidad o frecuencia de carga sobre la vida a fatiga de mezclas asfálticas se relaciona con la evolución de la rigidez. Así, en mezclas sometidas a ensayos de fatiga bajo el modo de carga de esfuerzo controlado, si la rigidez de la mezcla aumenta la vida a fatiga incrementa [12-14]. Un comportamiento contrario se reporta para el caso de mezclas sometidas al mismo ensayo pero bajo el modo de carga de deformación controlada [15-17]. Para el caso del presente estudio se analizará la respuesta que experimentan en ensayos de laboratorio la rigidez y la resistencia a fatiga de una mezcla asfáltica cuando se disminuye la frecuencia de carga simulando el efecto de la disminución de la velocidad vehicular que está ocurriendo actualmente en la ciudad de Bogotá D.C. sobre capas asfálticas gruesas.

\section{METODOLOGÍA}

\section{Caracterización de materiales y diseño de mezclas asfálticas}

Al agregado pétreo empleado para la elaboración de las mezclas asfálticas se le realizaron los ensayos requeridos para caracterizarlo, siguiendo las especificaciones del Instituto Nacional de Vías [18]. Los resultados de estos ensayos se presentan en la Tabla 1 y se observa que los valores cumplen con los requisitos mínimos de calidad exigidos por las especificaciones INVIAS [7] para fabricar mezclas tipo MDC-2 para capas de rodadura y tránsito NT3 (altos volúmenes).

Al cemento asfáltico CA 80-100 (PG 52-28) se le realizaron los ensayos típicos que exige la especificación INVIAS [7] para caracterizarlo (ver Tabla 2). Luego de realizar los ensayos al agregado pétreo y al CA se fabricaron cinco briquetas (compactadas a 75 golpes por cara) por cada porcentaje de asfalto entre 4,5\% y $6,5 \%$, con el fin de realizar el diseño Marshall (INV. E-748, INVIAS [18]) para determinar el contenido óptimo de asfalto de la mezcla. Para cumplir con las especificaciones del INVIAS [7] y fabricar mezclas asfálticas tipo MDC-2, se modificó la granulometría original de los agregados, tomando como referencia los valores 
promedios en porcentajes de la franja granulométrica que exige la especificación para la elaboración de las briquetas del ensayo Marshall (ver Tabla 3). Con los cálculos obtenidos del ensayo Marshall se escogió un porcentaje óptimo de CA de 5,3\%. En este porcentaje se están cumpliendo los requisitos mínimos exigidos por la especificación INVIAS [7] para MDC-2 y tránsitos tipo NT1 y/o NT2 (bajos y medios volúmenes de tránsito).

Tabla 1. Caracterización de los agregados.

\begin{tabular}{|l|c|c|c|}
\hline \multicolumn{1}{|c|}{ Ensayo } & Norma & Exigido & Resultado \\
\hline Peso específico & INV. E-223 & - & 2,56 \\
\hline $\begin{array}{l}\text { Equivalente de } \\
\text { arena }\end{array}$ & INV. E-133 & $50 \%$ mín. & $86 \%$ \\
\hline $\begin{array}{l}\text { Caras fracturadas } \\
\text { una cara }\end{array}$ & INV. E-227 & $85 \%$ mín. & $92 \%$ \\
\hline $\begin{array}{l}\text { Índice de } \\
\text { alargamiento }\end{array}$ & INV. E-230 & $10 \%$ máx. & $10 \%$ \\
\hline $\begin{array}{l}\text { Índice de } \\
\text { aplanamiento }\end{array}$ & INV. E-230 & $10 \%$ máx. & $10 \%$ \\
\hline $\begin{array}{l}\text { Ataque en } \\
\text { sulfato de } \\
\text { magnesio }\end{array}$ & INV. E-220 & $18 \%$ máx. & $17,4 \%$ \\
\hline $\begin{array}{l}\text { Desgaste } \\
\text { microdeval }\end{array}$ & INV. E-238 & $20 \%$ máx. & $20,3 \%$ \\
\hline $\begin{array}{l}\text { Resistencia al } \\
\text { desgaste } \\
\text { máquina de los } \\
\text { Ângeles }\end{array}$ & INV. E-218 & $25 \%$ máx. & $22,5 \%$ \\
\hline
\end{tabular}

Tabla 2. Características generales del cemento asfáltico CA 80-100.

\begin{tabular}{|l|c|c|c|c|}
\hline \multicolumn{1}{|c|}{ Ensayo } & Método & Unidad & CA & Resultado \\
\hline \multicolumn{4}{|c|}{ Ensayos sobre el asfalto original } \\
\hline $\begin{array}{l}\text { Penetración } \\
\left(25^{\circ} \mathrm{C}, 5 \mathrm{~s}\right)\end{array}$ & $\begin{array}{c}\text { ASTM } \\
\text { D-5 }\end{array}$ & $0,1 \mathrm{~mm}$ & $80-100$ & 85 \\
\hline $\begin{array}{l}\text { Índice de } \\
\text { penetración }\end{array}$ & $\begin{array}{c}\text { INV. } \\
\text { E-724 }\end{array}$ & - & $-1 /+1$ & $-0,5$ \\
\hline $\begin{array}{l}\text { Viscosidad } \\
\left(60^{\circ} \mathrm{C}\right)\end{array}$ & $\begin{array}{c}\text { ASTM } \\
\text { D-4402 }\end{array}$ & Poises & $\begin{array}{c}1.000 \\
\text { mín. }\end{array}$ & 1.400 \\
\hline $\begin{array}{l}\text { Ductilidad } \\
\left(25^{\circ} \mathrm{C}, 5 \mathrm{~cm} /\right.\end{array}$ & $\begin{array}{c}\text { ASTM } \\
\text { min })\end{array}$ & $\mathrm{D}-113$ & $\begin{array}{c}100 \\
\text { mín. }\end{array}$ & $>105$ \\
\hline $\begin{array}{l}\text { Solubilidad en } \\
\text { tricloroetileno }\end{array}$ & $\begin{array}{c}\text { ASTM } \\
\text { D-2042 }\end{array}$ & $\%$ & $\begin{array}{c}99 \\
\text { mín. }\end{array}$ & $>99$ \\
\hline $\begin{array}{l}\text { Contenido de } \\
\text { agua }\end{array}$ & $\begin{array}{c}\text { ASTM } \\
\text { D-95 }\end{array}$ & $\%$ & $\begin{array}{c}0,2 \\
\text { máx. }\end{array}$ & $<0,2$ \\
\hline $\begin{array}{l}\text { Punto de } \\
\text { inflamación } \\
\text { COC }\end{array}$ & $\begin{array}{c}\text { ASTM } \\
\text { D-92 }\end{array}$ & ${ }^{\circ} \mathrm{C}$ & $\begin{array}{c}232 \\
\text { mín. }\end{array}$ & 295 \\
\hline \multicolumn{4}{|c|}{ Ensayos sobre el residuo luego del RTFOT } \\
\hline $\begin{array}{l}\text { Pérdida de } \\
\text { masa }\end{array}$ & $\begin{array}{c}\text { ASTM } \\
\text { D-2872 }\end{array}$ & $\%$ & $\begin{array}{c}1,0 \\
\text { máx. }\end{array}$ & 0,2 \\
\hline $\begin{array}{l}\text { Penetración } \\
\left(25{ }^{\circ} \mathrm{C}, 5 \text { s }\right)\end{array}$ & $\begin{array}{c}\text { ASTM } \\
\text { D-5 }\end{array}$ & $\%$ & $\begin{array}{c}48 \\
\text { mín. }\end{array}$ & 65 \\
\hline
\end{tabular}

Tabla 3. Granulometría mezcla MDC-2.

\begin{tabular}{|c|c|c|}
\hline \multicolumn{2}{|c|}{ Tamiz } & \% que pasa \\
\hline Normal & Alterno & MDC-2 \\
\hline $19,0 \mathrm{~mm}$ & $3 / 4 ”$ & 100 \\
\hline $12,5 \mathrm{~mm}$ & $1 / 2{ }^{\prime}$ & $80-95$ \\
\hline $9,5 \mathrm{~mm}$ & $3 / 8 ”$ & $70-88$ \\
\hline $4,75 \mathrm{~mm}$ & $\mathrm{~N}^{\circ} 4$ & $49-65$ \\
\hline $2,00 \mathrm{~mm}$ & $\mathrm{~N}^{\mathbf{o}} 10$ & $29-45$ \\
\hline $0,425 \mathrm{~mm}$ & $\mathrm{~N}^{\mathbf{o}} 40$ & $14-25$ \\
\hline $0,180 \mathrm{~mm}$ & $\mathrm{~N}^{\mathbf{o}} 80$ & $8-17$ \\
\hline $0,075 \mathrm{~mm}$ & $\mathrm{~N}^{\mathbf{0}} 200$ & $4-8$ \\
\hline
\end{tabular}

\section{Fase experimental}

En las metodologías de diseño mecanicistas de pavimentos (p.e., [10]) los principales parámetros mecánicos utilizados para el dimensionamiento de las estructuras son la rigidez bajo carga repetida, la vida a fatiga y la deformación admisible a tracción de la mezcla. Para evaluar estos parámetros, luego de realizar el diseño Marshall se elaboraron las briquetas de mezcla asfáltica tipo MDC-2 necesarias para realizar ensayos de módulo resiliente y fatiga. El módulo resiliente (INV. E-749, INVIAS [18]) se midió bajo tres temperaturas $\left(10,20\right.$ y $\left.30^{\circ} \mathrm{C}\right)$ y frecuencias de carga $(2,5 ; 5,0$ y $10,0 \mathrm{~Hz})$ utilizando un equipo Nottingham Asphalt Tester (NAT). Con los resultados de estos ensayos se desarrolló una ecuación empírica para predecir la evolución de la rigidez de la mezcla analizada con la temperatura y la frecuencia de aplicación de carga. Para evaluar el cambio que experimenta la vida a fatiga de mezclas de concreto asfáltico con la rigidez se analizaron 360 ensayos de fatiga y módulo resiliente realizados sobre muestras de mezclas tipo MDC-1, MDC-2 y MDC-3 (acordes con INVIAS [7]) fabricadas con CA 80-100. Los resultados de las fatigas analizadas fueron obtenidos realizando ensayos por el método de tensión indirecta en un equipo NAT y siguiendo el procedimiento establecido por la especificación EN 12697-24E [19]. La temperatura del ensayo y la frecuencia de carga fueron de $20{ }^{\circ} \mathrm{C}$ y $2,5 \mathrm{~Hz}$ respectivamente. El tipo de carga ejecutado en el ensayo fue el de esfuerzo controlado. Este tipo de carga fue el utilizado teniendo en cuenta que en vías con medios y altos volúmenes de tránsito las capas asfálticas dimensionadas presentan espesor mayor a $10 \mathrm{~cm}$ experimentando este modo de carga [13-14, 20]. Las dimensiones de las muestras para los ensayos de fatigas son las mismas de las briquetas Marshall. Con los resultados de esta fase se desarrollaron dos ecuaciones empíricas que relacionan el cambio que 
experimenta la amplitud máxima de deformación admisible en tracción y la vida a fatiga de mezclas de concreto asfáltico con la rigidez.

Utilizando las ecuaciones desarrolladas se determinó, en laboratorio, el cambio en la resistencia a fatiga que experimenta una mezcla tipo MDC-2 cuando disminuye la frecuencia de aplicación de carga (la cual puede ser correlacionada con la velocidad de circulación vehicular).

\section{RESULTADOS}

En la Figura 1 se presenta la evolución del módulo resiliente $(E)$ de la mezcla MDC-2 con la temperatura $\left(T\right.$, en $\left.{ }^{\circ} \mathrm{C}\right)$ y la frecuencia de carga $(F$, en $\mathrm{Hz})$. Se observa un incremento típico del módulo cuando se aumenta la frecuencia de carga y disminuye la temperatura del ensayo. Esta evolución puede ser representada matemáticamente a través de la ecuación empírica (1). Para emplear esta ecuación es necesaria la identificación de seis variables de estado obtenidas a través de regresiones. Para el caso de la mezcla analizada los valores de estas variables se presentan en la Tabla 4. Estas variables no presentan un significado físico definido y deben cambiar principalmente con el tipo de ligante, la granulometría y el tipo de agregado pétreo utilizado para la fabricación de las mezclas. La ecuación (1) es válida para un rango de temperatura entre 10 a $30^{\circ} \mathrm{C}$ y frecuencias de carga de 0 a $10 \mathrm{~Hz}$ (velocidad aproximada de vehículos entre 0 a $70 \mathrm{~km} / \mathrm{h}$ ).

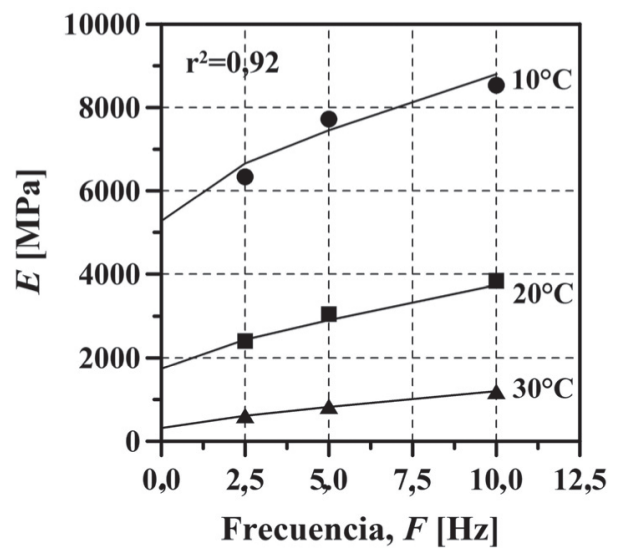

Figura 1. Evolución del módulo resiliente inicial $\left(E_{o}\right)$ de la mezcla MDC-2 con la temperatura (T) y la frecuencia de carga $(F)$.

$$
E=\exp \left(a+b T^{2}\right)+\left[\exp (c+d \sqrt{T})+\left(e+f T^{3}\right) \sqrt{F}\right]^{2}
$$

Tabla 4. Variables para simular la rigidez de la mezcla MDC-2 en función de $T$ y $F$.

\begin{tabular}{|c|c|c|c|c|c|}
\hline $\boldsymbol{a}$ & $\boldsymbol{b}$ & $\boldsymbol{c}$ & $\boldsymbol{d}$ & $\boldsymbol{e}$ & $\boldsymbol{f}$ \\
\hline 8,78 & $-0,0035$ & 5,26 & $-0,63$ & 12,4 & $-1,75 \mathrm{e}-4$ \\
\hline
\end{tabular}

La Figura 2 presenta los resultados de los ensayos de fatiga realizados sobre 360 muestras de concreto asfáltico tipo MDC-1, MDC-2 y MDC-3. Se observa una disminución del valor de $\mathrm{e}_{\max }$ (amplitud de la deformación para que el material falle cuando se aplican $N_{f}$ ciclos de carga) a medida que incrementa el módulo resiliente de las mezclas $(E)$, lo que equivale a un estado en el cual el material está cambiando su comportamiento de dúctil a frágil por rigidización. Estos resultados pueden ser representados matemáticamente a través de la ecuación (2). Se escogió esta ecuación teniendo en cuenta que para un nivel muy pequeño de amplitud de deformación aplicado al material, el mismo puede soportar infinitas veces dicha amplitud sin que haya ruptura (concepto de límite de resistencia a la fatiga, [21-22]). Los valores de las variables de estado de la ecuación (2) fueron determinados por regresión: $k_{1}=6,24 \times 10^{-6}, k_{2}=0,48$ y $k_{3}=15,79$. Resultados similares a los encontrados en este estudio fueron reportados por Schmidt y Santucci [15]. A pesar de que el material admite menores deformaciones en tracción a medida que se rigidiza, la vida a fatiga o el número de ciclos para que el mismo falle $\left(N_{f}\right)$ aumenta tal como se describe en la ecuación (3) (ver Figura 3). Estos resultados son acordes con la amplia evidencia experimental reportada en la literatura de referencia (p.e., [17, 23]).

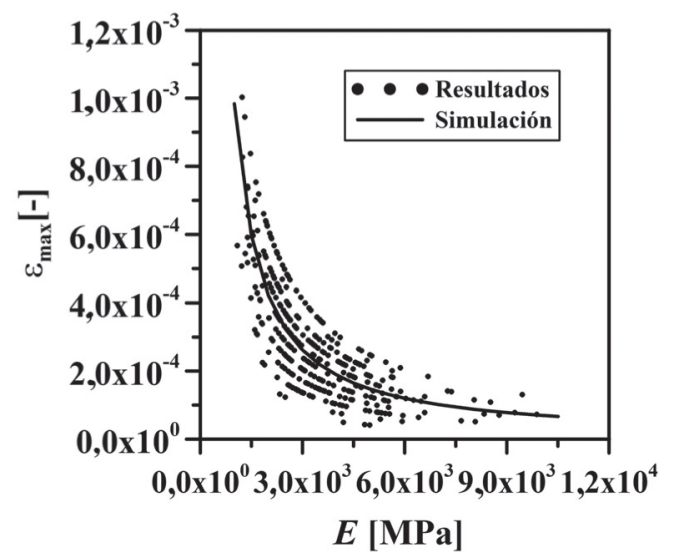

Figura 2. Evolución de $\varepsilon_{\max } \operatorname{con} E$. 


$$
\begin{gathered}
\varepsilon_{\max }=k_{1}+\frac{k_{2}}{E}+\frac{k_{3}}{E^{3 / 2}} \\
N_{f}=4,74\left(\frac{\sigma}{E}\right)^{-2,96} 10^{0,165}
\end{gathered}
$$

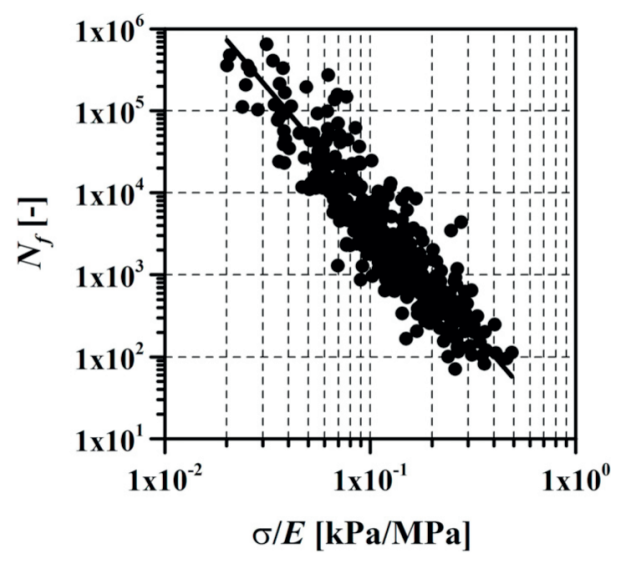

Figura 3. Evolución del número de ciclos de falla con $\sigma / E$.

En la Tabla 5 se presenta la evolución de la vida a fatiga de la mezcla analizada con el cambio en la velocidad de circulación vehicular. En la Figura 4 se presentan de manera esquemática los resultados presentados en la Tabla 5. El tiempo de aplicación de carga $t_{c}$ (en segundos) fue obtenido con base en la ecuación (4) recomendada por Lin [24-25], la cual genera resultados similares a aquellos reportados por Barksdale [26]. La frecuencia de carga fue obtenida de la ecuación (5) recomendada por Shell [11], la cual es la más utilizada en el mundo para su determinación [27]. Los parámetros $E, \varepsilon_{\max } \mathrm{y}$ $N_{f}$ se calcularon utilizando las ecuaciones (1-3) respectivamente. La temperatura $T$ utilizada para el cálculo de $E$ fue de $14{ }^{\circ} \mathrm{C}$ (temperatura media anual promedio reportada para Bogotá D.C.). El esfuerzo utilizado para el cálculo de $N_{f}$ fue de $662 \mathrm{kPa}$, el cual corresponde al aplicado por un eje simple de 13 toneladas, compuesto por 4 llantas, las cuales distribuyen la carga en un área circular de diámetro $25 \mathrm{~cm}$ (recomendado por [28-29]).

$$
\begin{gathered}
t_{c}=\frac{L}{V} 10^{0,004 h} \\
F=\frac{1}{2 \pi t_{c}}
\end{gathered}
$$

$L$ es la longitud de contacto de la llanta con el pavimento (generalmente de $30 \mathrm{~cm}$ ), $V$ es la velocidad del vehículo en $\mathrm{cm} / \mathrm{s}, h$ es la profundidad en $\mathrm{cm}$ a la cual se estima el $t_{c}$. En el presente estudio se supone un espesor de capa asfáltica de $20 \mathrm{~cm}$ (centro de la capa asfáltica, $h=10 \mathrm{~cm}$ ).

Tabla 5. Evolución de $N_{f}$ con la velocidad de circulación del vehículo $V$ para $\sigma=662$ $\mathrm{kPa}$.

\begin{tabular}{|c|c|c|c|c|c|}
\hline $\boldsymbol{V}[\mathrm{km} / \mathbf{h}]$ & $\boldsymbol{t}_{\boldsymbol{c}}[\mathrm{s}]$ & $\boldsymbol{F}[\mathrm{Hz}]$ & $\boldsymbol{E}[\mathrm{Mpa}]$ & $\mathrm{e}_{\max }[-]$ & $\boldsymbol{N}_{f}[-]$ \\
\hline 0,001 & $1.184,2$ & 0,00013 & $3.611,9$ & 0,000212 & 1.052 \\
\hline 5 & 0,237 & 0,67 & $4.058,51$ & 0,000187 & 1.485 \\
\hline 10 & 0,118 & 1,34 & $4.301,51$ & 0,000174 & 1.764 \\
\hline 15 & 0,079 & 2,02 & $4.510,19$ & 0,000165 & 2.030 \\
\hline 20 & 0,059 & 2,69 & $4.701,10$ & 0,000157 & 2.295 \\
\hline 25 & 0,047 & 3,36 & $4.880,65$ & 0,000151 & 2.564 \\
\hline 30 & 0,039 & 4,03 & $5.052,14$ & 0,000145 & 2.840 \\
\hline 50 & 0,024 & 6,72 & $5.687,91$ & 0,000127 & 4.034 \\
\hline 60 & 0,020 & 8,06 & $5.986,36$ & 0,000121 & 4.693 \\
\hline 70 & 0,017 & 9,41 & $6.276,17$ & 0,000114 & 5.398 \\
\hline
\end{tabular}

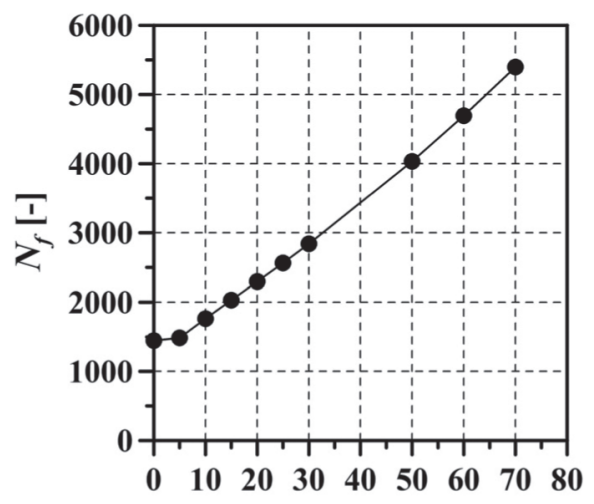

Velocidad $[\mathrm{km} / \mathrm{h}]$

Figura 4. Evolución de $N_{f}$ con $V$ para $\sigma=662 \mathrm{kPa}$.

Con los resultados obtenidos se reporta que la vida a fatiga de la mezcla analizada disminuye en laboratorio entre 39,5 y $77,6 \%$ cuando se reduce la velocidad de circulación desde $60 \mathrm{~km} / \mathrm{h}$ hasta 30 y $0 \mathrm{~km} / \mathrm{h}$ respectivamente, mientras que el módulo resiliente disminuye entre $15,6 \%$ y $39,7 \%$ respectivamente. Los valores obtenidos en el laboratorio de vida a fatiga tal como se observa en la Tabla 5 son pequeños. En campo las mezclas asfálticas tienden a experimentar mayor vida a fatiga tal como ha sido reportado ampliamente por diversos investigadores. Las diferencias entre las vidas a fatiga medidas en 
laboratorio e in situ se correlacionan a través de unos coeficientes denominados en la literatura de referencia como "shift factor". El Instituto del Asfalto [9], por ejemplo, recomienda un factor de 20, Finn, Saraf, Kulkarni, Nair, Smith y Abdullah [30] sugirieron un factor de 13,03, Brunton [31] de 440, Pell [32] entre 5 a 700, el estado de Washington en USA utiliza un rango entre 4 a 10 [33] y Castro y Sánchez [34] entre 10 y 100 . Futuras investigaciones se deben ejecutar con el fin de estimar la magnitud del "shift factor" para las mezclas de concreto asfáltico analizadas.

Si la temperatura $T$ simulada fuera de $19{ }^{\circ} \mathrm{C}$ (temperatura máxima de Bogotá D.C.), la disminución de la velocidad desde $60 \mathrm{~km} / \mathrm{h}$ hasta $30 \mathrm{~km} / \mathrm{h}$ y 0 $\mathrm{km} / \mathrm{h}$ generaría una disminución en la rigidez de la mezcla asfáltica de $19,4 \%$ y $47,4 \%$ respectivamente, lo que genera un decrecimiento en la vida a fatiga aproximado entre $47,2 \%$ y $85,1 \%$. Para el caso de $T=5{ }^{\circ} \mathrm{C}$ (temperatura mínima de Bogotá D.C.), los rangos de disminución de la rigidez y la vida a fatiga oscilan entre $12,5 \%-35,7 \%$ y $32,6 \%-73 \%$ respectivamente.

\section{CONCLUSIONES}

En este estudio se ejecutó una fase experimental destinada a medir la influencia de la disminución de la velocidad vehicular sobre las propiedades dinámicas de una mezcla de concreto asfáltico tipo MDC-2. El caso de estudio que se reporta es para mezclas MDC-2 que se fabrican y construyen en Bogotá D.C. Con los resultados obtenidos se concluye que la vida a fatiga obtenida en laboratorio, de este tipo de mezcla, disminuye entre 39,5 y $77,6 \%$ cuando se reduce la velocidad de circulación vehicular desde $60 \mathrm{~km} / \mathrm{h}$ hasta 30 y $0 \mathrm{~km} / \mathrm{h}$ respectivamente. Lo anterior es debido a que la mezcla experimenta un decrecimiento en su rigidez bajo carga cíclica, entre $15,6 \%$ y $39,7 \%$, en este rango de disminución de velocidad. Esta conclusión debe ser tenida en cuenta para capas asfálticas gruesas (superiores a $10 \mathrm{~cm}$ ) en donde el modo de carga representativo en los ensayos de fatiga es el de esfuerzo controlado. Adicionalmente la temperatura ambiente simulada fue la media anual que se reporta en Bogotá D.C. $\left(14{ }^{\circ} \mathrm{C}\right)$.

Futuras investigaciones deben ser realizadas para obtener la correlación entre la vida a fatiga obtenida en laboratorio y aquella que experimentan mezclas asfálticas in situ.

\section{REFERENCIAS}

[1] I. Thomson y A. Bull. "La Congestión del Tránsito Urbano: Causas y Consecuencias económicas y sociales". Revista de la Cepal. No 76, pp. 109-121. Santiago, Chile. 2002.

[2] R. Cal y Mayor y J. Cárdenas. "Ingeniería de Tránsito-Fundamentos y Aplicaciones". Ed. Alfaomega, México D.F. 2009.

[3] Concejo de Bogotá D.C. "Proyecto de Acuerdo No 248 de 2009 por Medio del cual se Adiciona el Numeral 4 al Artículo 95 del Acuerdo 79 de 2003, y se Dictan otras Disposiciones". 5 de agosto de 2010. Fecha de consulta: 15 de diciembre de 2010. URL: http://www.alcaldiabogota.gov.co/sisjur/ normas/Norma1.jsp?i=36492

[4] Secretaría Distrital de Movilidad de Bogotá D.C. "Entrevista al Secretario de Movilidad de Bogotá D.C. Fernando Álvarez por el periódico El Tiempo". 3 de octubre de 2010. Fecha de consulta: 8 de enero de 2011. URL: http://www.eltiempo.com/archivo/buscar?q $=$ secretaria + de + movilidad $\& a=2010 \&$ pagin $\mathrm{a}=1 \& \mathrm{~m}=10$

[5] Secretaría Distrital de Movilidad de Bogotá D.C. "Contrato 966 de 2010 para la Toma de Información de Campo para el Programa de Monitoreo, Seguimiento y Planeación del Tránsito y el Transporte de Bogotá D.C.". Bogotá D.C., Colombia. 2010.

[6] Ministerio de Transporte de la República de Colombia. "Resolución 1384 de 2010, por el cual se Adopta el Método para Establecer los Límites de Velocidad en las Carreteras Nacionales, Departamentales, Distritales y Municipales de Colombia". 20 de abril de 2010. Fecha de consulta: 10 de junio de 2010. URL: http://www.alcaldiabogota.gov. co/sisjur/normas/Norma1.jsp?i=39357.

[7] INVIAS-Instituto Nacional de Vías. "Especificaciones Generales para Construcción de Carreteras". Bogotá D.C., Colombia. 2007.

[8] AASHTO-American Association of State Highway and Transportation Officials. "Guide for Design of Pavement Structures". Washington, D.C. 1993.

[9] Asphalt Institute-AI. "Research and Development of the Asphalt Institutes Thickness Design Manual”. MS 1, 9th Ed. College Park, Maryland, Estados Unidos. 1982. 
[10] MEPDG. ARA, Inc., ERES Consultants Division. "Guide for the MechanisticEmpirical Design of New and Rehabilitated Pavement Structures". NCHRP Project 1-37A, Transportation Research Board, Washington, D.C. URL: www.trb.org/mepdg/. Accessed: September 12 of 2007. 2004.

[11] Shell International Petroleum Company. "Shell Pavement Design Manual, Asphalt Pavement and Overlays for Road Traffic". London (U.K.). 1978.

[12] T.-W. Hsu and K.-H. Tseng. "Effect of Rest Periods on Fatigue Response of Asphalt Concrete Mixtures". Journal of Transportation Engineering. Vol. 122, Issue 4, pp. 316-322. 1996.

[13] M.A. Elseifi, G.W. Flintsch and I.L. AlQadi. "Quantitative Effect of Elastomeric Modification on Binder Performance at Intermediate and High Temperatures". Journal of Materials in Civil Engineering. Vol. 15, Issue 1, pp. 32-40. 2003.

[14] P. Baburamani. "Asphalt Fatigue Life Predictions Models-a Literature Review". Research Report ARR 334. 1999.

[15] R.J. Schmidt and L.E. Santucci. "The Effect of Asphalt Properties on the Fatigue Cracking of Asphalt Concrete on the Zaca-Wigmoretest Project". In: Proceedings, Association of Asphalt Paving Technologists. Vol. 38, pp. 39-64. 1969.

[16] J.A. Epps and C.L. Monismith. "Fatigue of Asphalt Concrete Mixtures-Summary of Existing Information. Fatigue of Compacted Bituminous Aggregate Mixtures". ASTM STP 508, American Society for Testing and Materials, pp. 19-45. 1972.

[17] H. Di Benedetto and C. De la Roche. "State of the Art on Stiffness Modulus and Fatigue of Bituminous Mixtures". Report RILEM $\mathrm{N}^{\circ} .17$ Bituminous Binders and Mixes: State of the Art and Interlaboratory Test on Mechanical Behavior and Mix Design, L. Francken, ed., E and FN Spon, London, pp. 97-123. 2005.

[18] INVIAS - Instituto Nacional de Vías. "Normas de Ensayos de Materiales para Carreteras". V. I y II. Bogotá D.C., Colombia. 2007.

[19] CEN-Committee European of Normalization. "Bituminous Mixture Test Methods for Hot Mix Asphalts". Part25: Cyclic Compression Tests. 2005.
[20] Y.H. Huang. "Pavement Analysis and Design". Second edition, Pearson Prentice Hall, Upper Saddle River, NJ. 2004.

[21] F.A. Reyes. "Diseño Racional de Pavimentos". Escuela Colombiana de Ingeniería, $1^{\text {ra }}$. Edición, Bogotá D.C., Colombia. 2003.

[22] S.H. Carpenter, K.A. Ghuzlan and S. Shen. "Fatigue Endurance Limit for Highway and Airport Pavement". Transportation Research Record (TRR): Journal of the Transportation Research Board. No 1832, pp. 131-138. TRB, National Research Council, Washington D.C. 2003.

[23] H. Di Benedetto, C. De la Roche, H. Baaj, A. Pronk and R. Lundstrom. "Fatigue of Bituminous Mixtures". Material and Structures. Vol. 37, Issue 4, pp. 202-216. 2004.

[24] X.X. Lin. "A Method of Determining the Relevant Parameters in the Calculation of Pavement Deformation of Bituminous Surface". China J. Highway and Transport. $\mathrm{N}^{\circ}$ 2, pp. 10-20. 1989.

[25] X.X. Lin. "Study on Semi-Rigid Asphalt Pavement". Rep. of Key Sci. \& Tech. Projects 85-65. Ministry of Communications. Beijing, China. 1989.

[26] R.D. Barksdale. "Compressive Stress Pulse Times in Flexible Pavements for Use in Dynamic Testing". Highway Research Record, 345, Highway Research Board, Washington, D.C., pp. 32-44. 1971.

[27] J.A. D'Angelo, R.N. Dongre and L.A. Myers. "Conversion of Testing Frequency to Loading Time: Impact on Performance Predictions Obtained from MechanisticEmpirical Pavement Design Guide". In: Transportation Research Board 85th Annual Meeting, TRB, p. 16. 2006.

[28] IDU-Instituto de Desarrollo Urbano y Universidad de los Andes. "Manual de Diseño de Pavimentos para Bogotá". Bogotá D.C., Colombia. 2002.

[29] H.A. Rondón y F.A. Reyes. "Pavimentos Flexibles". Editorial Académica Española, p. 481. Madrid, España, 2011. ISBN 978-3-8454-9906-2.

[30] F. Finn, C.L. Saraf, K. Kulkarni, K. Nair, W. Smith and A. Abdullah. "Development of Pavement Structural Subsystems". Final Report, Project 1-10B. 1977. 
[31] J.M. Brunton, S.F. Brown and P.S. Pell. "Development of the Analytical Design Method of Asphalt Pavements". In: Sixth International Conference on The Structural Design of Asphalt Pavements. Vol. 1 Proc. University of Michigan. Ann Arbor, Michigan, USA. July 13-17, 1987.

[32] P.S. Pell. "Keynote Lecture-Pavement Materials". In: 6th International Conference of Structural Design of Asphalt Pavements. University of Michigan. Ann Arbor, Michigan, USA, pp. 310-323. July 13-17, 1987.
[33] W.M. Nassar. "Analysis and Validation of the Mechanistic-Empirical Overlay Design Procedure Incorporated in FLEXWIN Computer Program". Master's Thesis. University of Idaho. Idaho, USA, p. 324. 1997.

[34] M. Castro and A.J. Sánchez. "Fatigue and Healing of Asphalt Mixtures: Discriminate Analysis of Fatigue Curves". Journal of Transportation Engineering. ASCE, Vol. 132, Issue 2, pp. 168-174. 2006. 